\title{
Numerical simulation of thermal-hydraulic generators running in a single regime
}

Topical Issue: AMMA 2013

\author{
Nicolae Chioreanu ${ }^{1}$, Tudor Mitran ${ }^{1 *}$, Alexandru Rus $^{1}$, Horia Beles $^{1}$ \\ 1 University of Oradea, Romania
}

Received 01 June 2013; accepted 17 December 2013

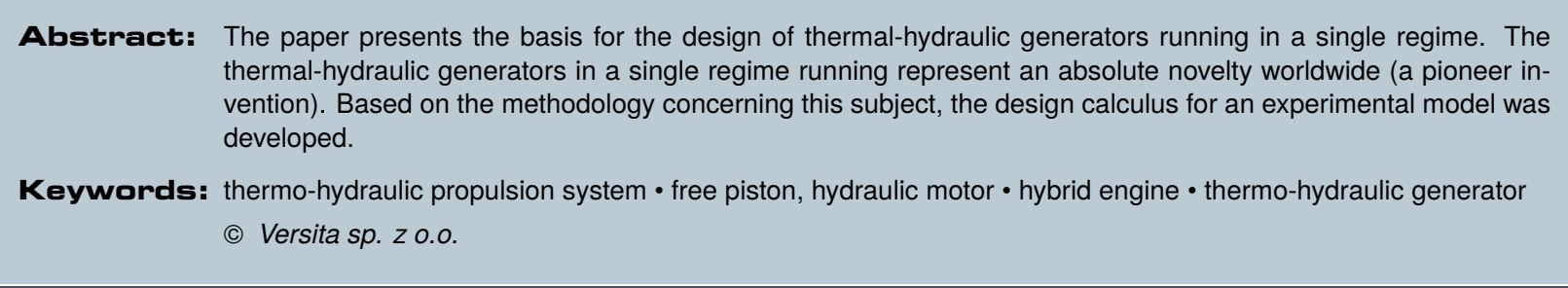

\section{Introduction}

This paper presents the design basis of the thermohydraulic generators with a single regime running.

The thermal-hydraulic generator in a single regime running is the main part of the new thermal-hydraulic propulsion systems. The new thermal-hydraulic system includes: a thermo-hydraulic generator (GTH), a hydro-pneumatic accumulator $(\mathrm{AH})$ and a hydraulic motor $(\mathrm{MH})$ (Figure 1). The propulsion system is provided also with a hydraulic liquid tank $(\mathrm{RH})[1,2]$.

The thermal-hydraulic generator transforms the thermal energy obtained by the burning of a fuel (gasoline, diesel fuel, unconventional fuels) into hydrostatic energy. The hydrostatic energy produced by the generator is stored in the hydro-pneumatic accumulator.

The thermal hydraulic generator is characterized by single regime running (constant injected fuel quantity in every

*E-mail: tudor_mitran@yahoo.com

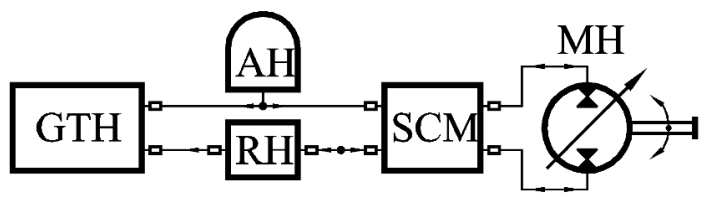

Figure 1. The scheme of the thermal-hydraulic propulsion system

engine cycle) and automatic functioning on the STARTSTOP principle that means there is an automatic start when the energy (pressure) in the accumulator has the minimum admissible value and an automatic stop when the energy pressure) in the accumulator has the maximum admissible value. The hydraulic accumulator is of hydro-pneumatic type. The accumulator has the role to accumulate the energy produced by the thermal-hydraulic generator that is necessary to run the resistant strokes of the engine cycle and to supply the hydraulic motor (motors). What is more, the accumulator has the role to dump 




Figure 2. New concept regarding the running of the propulsion systems

flow pulsations and hydraulic shocks.

The supply of the hydraulic motor (motors) with hydrostatic energy is realized through the engine's command system (SCM). The hydraulic motor (motors) transforms the hydraulic energy from the accumulator into mechanical energy necessary to run the vehicle (under the form of rotational movement). The hydraulic motor is a machine with a variable and reversible volumetric capacity. In this way the recovery of energy during breaking can be realized by retransforming it into hydrostatic energy stored in the accumulator. The running and the construction of the new thermal-hydraulic propulsion systems is based on a new concept that consists in the supply of the motor machine (MM) from an energy accumulator. Charging the energy accumulator is made by an energy generator that runs in a single regime, with maximum efficiency. The concept involves the development of propulsion systems, generally consisting of an energy generator (GE), an energy accumulator (AE) and a motor machine (MM). Two energy transformations that take place are the primary transformation and the secondary transformation (Figure 2) [3]. The primary transformation is realized by the energy generator, which transforms the input energy (thermal or electric) in another form of energy (different from the fluid energy). The energy produced by the generator is stored in the energy accumulator (AE). The secondary transformation is realized by the motor machine (MM), which takes over the energy stored in the accumulator and transforms it into mechanical energy, usually under the form of a rotation movement.

The propulsion systems runs in the following way: the generator (GE) transmits the produced energy to the accumulator $(A E)$, until the energy in the accumulator reaches its maximum admissible value. In this moment, the accumulator automatically stops running until the energy in the accumulator drops to the minimum admissible value/level. In this way, the generator (GE) can run in one regime only, with maximum efficiency, independently from the working regime of the motor machine (MM). The motor machine takes over the energy from the accumulator and transforms it into mechanic energy at the power necessary to run the working parts of the system. The energy flow can go in both directions generator-accumulator and reverse, or accumulator-motor machine and reverse.

The propulsion system runs efficiently if the energy gen- erator (GE) fulfils the following conditions: to produce a form of energy that can be easily stored in the energy accumulator $(\mathrm{AE})$ and can be easily transmitted to the motor machine $(\mathrm{MM})$; to run in a single regime, automatic, on the start-stop principle.

The energy forms, which fulfil the above mentioned conditions are a hydrostatic energy and a pneumatic energy. The hydrostatic energy can be used in a large scale of power and application domains. The hydrostatic drives are well known and considered to be superior compared to the other existing drives [4]. The pneumatic energy is used at low power. Generally, the pneumatic drives are used in restricted areas of activity.

Considering the energy form that we produce, generators are classified in: hydrostatic generators and pneumatic generators. The hydrostatic generators which transform the thermal energy are called thermal-hydraulic single regime generators, while those which transform the electric energy are called electro-hydraulic single regime generators. The energy accumulator is a normal hydraulic accumulator. The motor machine is a rotational hydraulic engine(s) with adjustable capacity. These types of engines allow for the variation of the rotational speed on a large scale and can recover the braking energy [6].

The new propulsion systems belong to the category of hybrid thermal-hydraulic or electro-hydraulic propulsion systems. They represent an absolute novelty. Hybrid engines are known as hybrid thermal-electric engines (hybrid series and parallel hybrid), which produce three energy conversions: thermal energy is transformed in mechanic energy (through the internal combustion engine); mechanic energy is transformed in electric energy (through the electric energy generator); electric energy is transformed into mechanic energy (through the electric engines) or it is stored in electric accumulators. At the new propulsion systems there are only two energy transformations that occur: the thermal or electrical energy is transformed into hydrostatic or pneumatic energy (through the energy generator); and the hydrostatic or pneumatic energy is transformed into mechanic energy (through hydraulic or pneumatic engines).

The new concept regarding the running and the construction of the new thermal-hydraulic propulsion systems in a single regime represents a new research direction. Obviously, it is much easier to optimize a single regime, compared to an infinity as it happens at the current engines. 


\section{The design methodology applied at a two stroke termo-hydraulic gener- ator [3-5]}

The main parts of a two stroke thermal-hydraulic generator with a uniflow scavenge with an exhaust valve are: a motor cylinder (CM) and a hydraulic cylinder $\mathrm{CH}$, coaxial assembled, and inside them the free piston (PL) has a reciprocated movement [1,2] (Figure 3). The free piston is the only mobile part, without any articulating elements. The start-stop of the piston at the end of the stroke does not negatively affect the functioning of the generator because the speed, the kinetic speed respectively, is zero at the ends of the stroke. Due to the piston movement, between the cylinder's walls and the piston, four chambers with variable volume are formed: a thermal chamber $(T)$, a compression chamber $(\mathrm{C})$ and hydraulic chambers $(\mathrm{H} 1)$ and $(\mathrm{H} 2)$. The piston displacement takes place under the action of the pressure forces produced by the gases inside the thermal and the compression chamber, respectively; and of the pressure forces produced by the hydraulic liquid in the chambers $(\mathrm{H} 1)$ and $(\mathrm{H} 2)$. In the thermal chamber the following processes of the engine cycle take place: intake, compression, burning, expansion and exhaust (exactly as in a Diesel engine). Whereas, in the compression chamber the intake process of the fresh air takes place.

In the hydraulic chambers pumping processes (suctiondischarge) of the hydraulic liquid occur. The piston movement is coordinated by the automatic command and control system (SAC). The information regarding the piston position is provided by the position sensors (D1), (D2) and (D3). The piston stroke is between the point of minimum volume (pvm) and the point of maximum volume (pvM). The forces acting on the piston are: the force developed by the gas pressure inside the thermal and the compression chamber, and force developed by the hydraulic liquid pressure inside the chambers $(\mathrm{H} 1)$ and $\left(\mathrm{H}_{2}\right)$. During the piston stroke, the force developed by the gas pressure inside the thermal chamber has a large variation (exponential) and the force developed by the liquid pressure inside the chambers $(\mathrm{H} 1)$ and $(\mathrm{H} 2)$ is almost constant.

The hydraulic energy stored in the accumulator during an engine cycle is determined by the following relation [3-5]:

$$
W_{H}=\eta_{t p} \times \oint\left[p_{T}(x)-p_{C}(x)\right] \times d V[J]
$$

where: $\eta_{t p}$ is the total efficiency of the generator, $p_{T}(x)$ is the function for the variation of the gases pressure inside the thermal chamber, $p_{C}(x)$ is the function for the variation of the gas pressure inside the compression chamber, $x$ is the position of the piston regarding the origin point of the

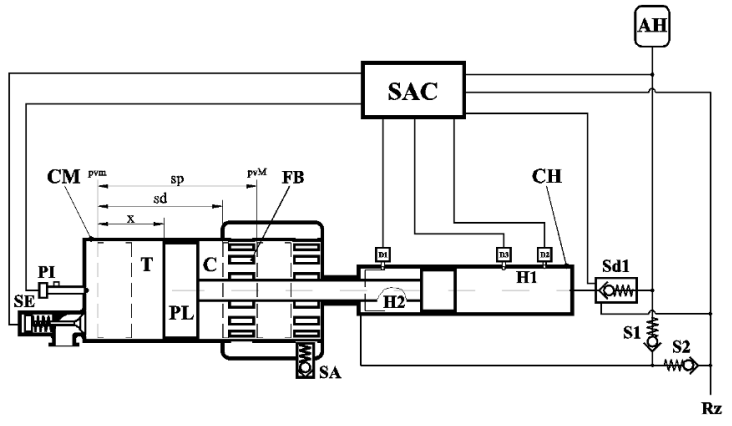

Figure 3. The scheme of the uniflow scavenge two strokes thermogenerator

coordinate axis.

The value of the total efficiency of the thermo-hydraulic generator is a function of the energy losses that occur during an engine cycle: energy losses produced by the friction forces, energy losses produced by the hydraulic liquid viscosity, etc.

The power developed by the thermo-hydraulic generator is determined on the basis of the following relation:

$$
P_{H}=f_{c t} \times W_{H}=P_{h a} \times Q_{p}[k W]
$$

where: $f_{c t}$ is the frequency of the engine's cycles, pha is the pressure of the liquid inside the accumulator and $\mathrm{Qp}$ is the flow of the single running regime generator.

By solving the system of equations (1) and (2), the following parameters are obtained: the engine cylinder's inner diameter $\mathrm{D}$, the piston displacement $\mathrm{Sp}$ and the minimum volume of the thermal chamber. The kinetic energy $\mathrm{Ec}(\mathrm{x})$ developed by the piston PL during its movement until the point $\mathrm{x}$ is defined by the following function:

$$
E c(x)=L g(x)=c L h(x)[J]
$$

where: $\operatorname{Lg}(x)$ is the mechanical work developed by the gas pressure inside the engine's cylinder until the point $x, \operatorname{Lh}(x)$ is the mechanical work developed by the liquid pressure inside the hydraulic cylinder and $c$ is a coefficient.

The value of the coefficient $c$ depends on the movement sense of the piston PL and on the value of the mechanical efficiency. The mechanical efficiency is reported to the mechanical work produced by the hydraulic cylinder $\eta_{m}=1-|L f(x) / L h(x)|$ and it evaluates the friction and the hydraulic liquid viscosity forces. If the sense of the piston movement coincides with the coordinate axis sense (the useful stroke) than: $c=2-\eta_{m}$, and if it opposites: $c=\eta_{m}$. 
At the ends of the piston stroke, the kinetic energy is zero: $E c(x)=0 ; E c(s p)=0$.

If the theory of the kinetic energy is applied to the two strokes of the piston, the diameter of the hydraulic cylinder and the one of the rod can be obtained (the rod is the connecting part between the engine piston and the hydraulic piston).

The function for the variation of the piston speed is given by the following relation:

$$
w p(x)=\sqrt{\frac{2 \times E c(x)}{m_{p}}}[\mathrm{~m} / \mathrm{s}]
$$

where: $\mathrm{mp}$ is the mass of the free piston (PL)

The function for the variation of the piston acceleration is given by the following relation:

$$
a p(x)=\frac{d}{d \tau} w p(x)=w p(x) \times \frac{d}{d x} w p(x)\left[m / s^{2}\right]
$$

The piston mass is determined from the condition of limiting the maximum speed of the piston during the expansion stroke to a certain value wp max. The maximum speed is reached in the point $x a$, where the piston acceleration is zero:

$$
a_{p d}\left(x_{a}\right)=0\left[\mathrm{~m} / \mathrm{s}^{2}\right]
$$

The solution of the equation (6) represents the value of the coordinate $x_{a}, w_{p \max }=m p\left(x_{a}\right)$.

If the theorem of the kinetic energy is applied between the point $x=0(p v m)$ and the point $x_{a}$, the relation for the calculus of the piston mass is obtained:

$$
m_{p}=\operatorname{frac} 2 \times E c\left(x_{a}\right) w_{p \max }^{2}[k g]
$$

The time in which a piston stroke $i$ takes place is determined with the following relation:

$$
\tau_{p i}=\frac{s_{p}}{\bar{w}_{p i}}=\frac{s_{p}^{2}}{\int_{x_{0}}^{x_{0}+s_{p}} w_{p i}(x) \times d x}[s]
$$

where: $w_{p i}(x)$ is the function for the variation of the piston speed in stroke $\mathrm{i}$ and $\bar{w}_{p} i$ is the average speed of the piston in stroke $i$. The frequency of the engine cycles is calculated according to the following relation:

$$
f_{c t}=\frac{1}{\sum_{i=1}^{2} \tau_{p i}}[H z]
$$

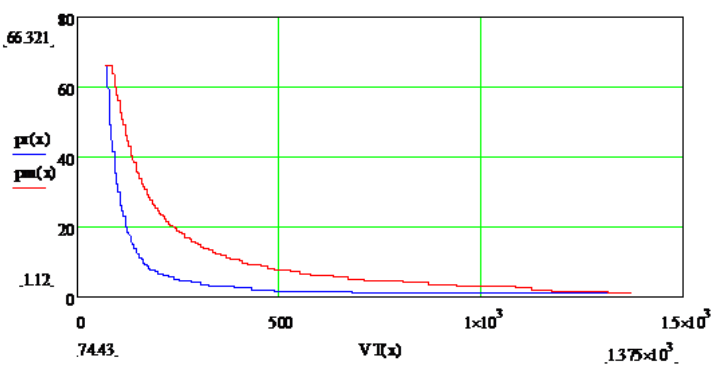

Figure 4. The indicated diagram for the thermal chamber

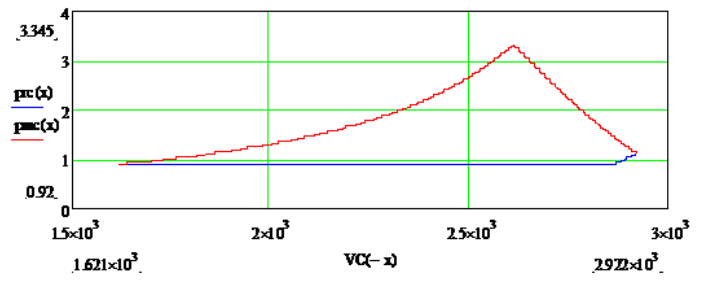

Figure 5. The indicated diagram for the compression chamber

\section{Numerical approach}

Below the data obtained by calculus during the design of the experimental model of the thermal-hydraulic generator are presented. The initial data for the design are: the power $\mathrm{Pp}=(15-20) \mathrm{kW}$; the flow $\mathrm{Qp}=(1,5-2) \mathrm{l} / \mathrm{s}$ and the mean pressure in the accumulator is $p h=10,5 \mathrm{MPa}$. After the thermal calculus, the following parameters were obtained: the maximum volume of the thermal chamber $V_{b}=1375 \mathrm{~cm} 3$, the minimum volume of the burning chamber $V_{v}=74,43 \mathrm{~cm} 3$, the expansion volume $V_{d}=1064 \mathrm{~cm} 3$, the compression volume $\mathrm{V}_{a}=778,68 \mathrm{~cm} 2$, the total stroke $\mathrm{s}_{p}=142 \mathrm{~mm}$, the piston displacement in the expansion stroke $\mathrm{s}_{d}=108 \mathrm{~mm}$, the compression stroke $\mathrm{s}_{a}=85 \mathrm{~mm}$, the bore $\mathrm{D}=108 \mathrm{~mm}$, the ports dimensions $\mathrm{hf} \times \mathrm{g}_{f}=28 \times 4 \mathrm{~mm}$, the number of the ports $\mathrm{n}_{f}=18$, the maximum gas pressure $\mathrm{p}_{y}=6,63 \mathrm{MPa}$, the gas pressure at the end of the expansion process $\mathrm{p}_{d}=0,31 \mathrm{MPa}$, the injected fuel mass/cycle $\mathrm{m}_{c b}=4,07 \cdot 10^{-5} \mathrm{~kg}$. The indicated diagram in the thermal chamber $(\mathrm{T})$ is presented in Figure 4.

The indicated diagram for the compression chamber $(\mathrm{C})$ is presented in Figure 5.

Numerical values obtained after the calculus of the free piston $(\mathrm{PL})$ : Initial data: the mechanical efficiency: $\eta_{m}=0,975$, the piston maximum speed: $\mathrm{w}_{\operatorname{pmax}}=12 \mathrm{~m} / \mathrm{s}$ The calculus of the dimensional parameters: the diameter of the engine piston head $D=108 \mathrm{~mm}$, the diameter of the hydraulic piston head $\mathrm{d}_{h}=\mathrm{D}_{h}=30 \mathrm{~mm}$, the rod diameter $\mathrm{d}_{t}=18 \mathrm{~mm}$, the free piston mass $\mathrm{m}_{p}=7,95 \mathrm{~kg}$. The calcu- 


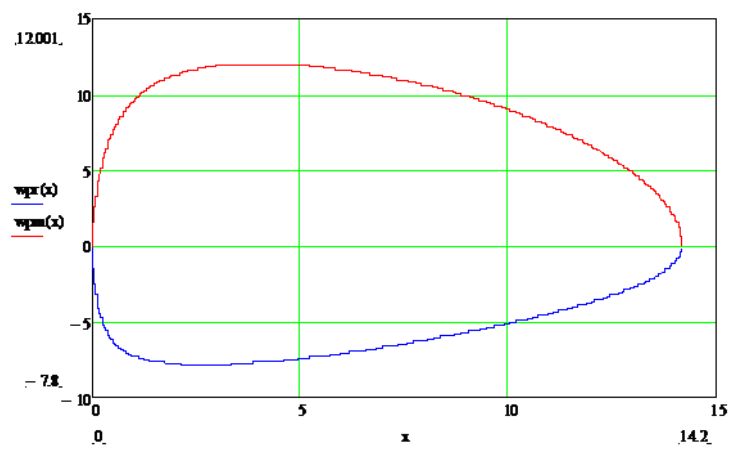

Figure 6. The variation of the piston speed

lus of the kinematic parameters: the average speed of the piston during the compression stroke $\mathrm{w}_{m r}=5,8 \mathrm{~m} / \mathrm{s}$, the movement time during the compression stoke $\tau_{p r}=0,024$ $\mathrm{s}$, the average speed during the useful stroke $\mathrm{w}_{m m}=9,3$ $\mathrm{m} / \mathrm{s}$, the movement time in the useful stroke $\tau_{p m}=0,015 \mathrm{~s}$, the average speed during one engine cycle $\mathrm{w}_{m}=7,2 \mathrm{~m} / \mathrm{s}$, the movement time during one engine cycle $\tau_{p m}=0,04 \mathrm{~s}$, the frequency of the engine cycle $f_{p}=25,2 \mathrm{~Hz}$. The piston speed variation is presented in Figure 6.

The calculus of the energetic parameters: The power $\mathrm{P}_{p}=16,57 \mathrm{~kW}$, the mean flow of the hydraulic liquid $\mathrm{Q}_{p}=1,59 \mathrm{l} / \mathrm{s}$, the mean pressure of the hydraulic liquid $\mathrm{p}_{h}=105$ bar

\section{Results and discussion}

The new concept regarding the development of propulsion system is a new research direction that allows for the manufacturing of new types of propulsion systems with improved performances and with low fuel consumption and emissions [3]. For example, in the case of road vehicles equipped with the new thermo-hydraulic propulsion system the mechanical transmission (clutch, gear box, longitudinal transmission) will be partially eliminated if the system has only one hydraulic motor, or will be totally eliminated if the system has two or four hydraulic motors. The breaking of the vehicle is realized when the hydraulic motors are converted into a pump regime (an efficient breaking can be realized, to the limit of the adherence, without blocking the wheels) and with breaking energy recovery. Furthermore, adjustment systems (the electronic system of the power train, the electronic command of the gear box, etc.) and the start system are eliminated [3]. In conclusion, the new vehicles equipped with the new propulsion system willperform at least equal with those of the current vehicles equipped with automatic transmission and, obviously, with greater energetic efficiency (by running in a

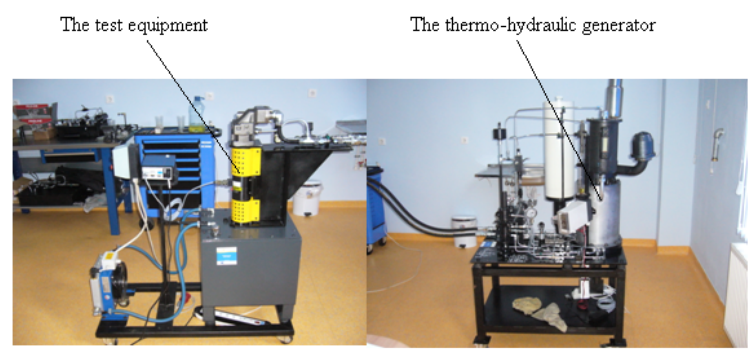

Figure 7. The experimental model of the thermo-hydraulic generator

single regime the optimization of the functioning is easier, without being in idle and with the possibility of breaking energy recovery). The thermal part of the propulsion system (the thermal-hydraulic generator) is characterized by the particularities of single regime running (no idle). It is estimated that, compared with the nowadays propulsion systems, the new thermal-hydraulic system has the following advantages: lower fuel specific consumption and emissions (it is easier to optimize one regime instead of an infinity); simple construction; and greater reliability. On the basis of the computer model developed, an experimental model was built (see Figure 7).

\section{ACKNOWLEDGMENTS}

This paper is a research result of the project "Research on engines in a single regime running," financed with European funds through the Hungary-Romanian Cross-Border Cooperation Program 2007-2013.

\section{References}

[1] Chioreanu, N., Single regime thermal engines (in Romanian), University of Oradea Publishing House, Oradea, 2006.

[2] Chioreanu, N., Chioreanu Ş., Engines for nonconventional motor vehicles (in Romanian), University of Oradea Publishing House, Oradea, 2006.

[3] Chioreanu, N., Chioreanu S.., New concept for the running of engines. Proceedings of the Romanian Academy, Series A, Volume 11, Number 2/2010, pp. 148-155

[4] Chioreanu N., Mitran T., Bártfai Z., Lágymáyosi A. Basic principles for the design of the monoregime thermal engines. Proceedings of the annual session of scientific papers IMT Oradea 2012

[5] Chioreanu N., Mitran T., Bártfai Z., Blahunka Z. The main parameters of the monoregime thermal engines. 
Proceedings of the annual session of scientific papers IMT Oradea $2012 \breve{A}$

[6] Vasiliu N., Vasiliu D., Seteanu I., Rădulescu V., Fluid mechanics and hydraulic systems. Fundamentals and applications, Vol. II, (in Romanian), Technical Publishing House, Bucharest, 1999. 